\title{
LA INFORMACIÓN COMO UN PRODUCTO, LA COMUNICACIÓN COMO UN DERECHO
}

\author{
INFORMATION AS A PRODUCT, \\ COMUNICATION AS A RIGHT
}

Amelia Ribadeneira Universidad Internacional del Ecuador - Ecuador

María Paula Romo

Universidad Internacional del Ecuador - Ecuador

Ph. D. Diego Donoso V. Universidad Tecnológica Israel - Ecuador ddonoso@uisrael.edu.ec

Fecha de recepción: 15/04/2016

Fecha de aceptación: 18/05/2016

\begin{abstract}
Resumen
La comunicación al ser un acto intrínseco de las personas no puede ser considerada como un bien público, porque comunicar es cualquier acto que realiza un ser humano a través de las palabras, las señas o el silencio. En cambio, la información es un producto de consumo inmaterial por parte de los ciudadanos. Los medios masivos transmiten información que puede afectar a los poderes que están en disputa.
\end{abstract}

Palabras Claves: Comunicación, Información, Ciudadanía 


\section{Abstract}

Communication being an intrinsic act of the people and can not be considered as a public good, because communicating is any act performed by a human being through words, signs or silence. On the other hand, information is a product of intangible consumption by citizens. The mass media transmit information that may affect the powers that are in dispute.

Keywords: Communication, Information, Citizenship 


\section{Introducción}

La comunicación nace con la vida. Son los seres humanos, de entre todos los animales, quienes más la desarrollan y utilizan para su evolución. La comunicación es pensamiento, es creación, es herramienta, es arte, es acción, es negocio. La comunicación tiene dos dimensiones, una humana, interpersonal, que puede ser entendida en el mundo de lo no transaccional; y otra pública, que permite analizarla en el contexto de la industria, de la política, de la ideología, de las finanzas, de la cultura. No obstante, ambas están interrelacionadas, porque dependen de la intervención humana para su realización.

Mattelart (1997: 13) relata que la comunicación fue "el centro de la ideología del progreso" en el siglo XVIII, los ingleses la consideraron una contribución para organizar el trabajo colectivo dentro de la fábrica y para estructurar los espacios económicos. Los franceses añadieron a la visión del progreso "la realización de la razón". En la primera mitad del siglo XIX, durante la era industrial, se concibe a la comunicación como un sistema que funciona en red, lo que equivale a la conexión del transporte y las finanzas.

Luego, en la segunda mitad del mismo siglo, se plantea a la comunicación como un sistema orgánico similar al cuerpo humano, en el que todo está interconectado: "una sociedad-organismo cada vez más coherente e integrada, donde las funciones son cada vez más definidas y las partes cada vez más interdependientes" (Mattelart, 1997: 16).

En Historia de las teorías de la Comunicación, Mattelart reseña que a finales del siglo XIX surgen la sociedad de masa y los medios masivos de comunicación, estos últimos como principales constructores de opinión. También nacen los lectores, la muchedumbre hipnotizada e irreflexiva, que a mediados del siglo XX sube al escalafón de público.

A diferencia de lo que sucedió en los siglos XVIII y XIX, en que la comunicación estuvo más entendida en relación con el trabajo, los negocios y las finanzas, los estudios del siglo XX apuntaron a la importancia de la comunicación a partir de su capacidad de distribuir masivamente los mensajes, es decir, al papel que desempeñaron los medios en cuanto a la ideología, a la política y a la cultura. Resumirlo así, no implica que la comunicación pierda su categoría de negocio, solamente se trata de diferenciar el peso que tuvo en cada proceso histórico.

Hay mucha razón al decir que el siglo XX fue el siglo de las comunicaciones, y ello se debe básicamente a cuatro factores: la política, la tecnología, la teoría y los derechos. Con la política está la relación con el poder, 
el estado y la ideología; con la tecnología están la economía y los negocios; con la teoría, los estudios, las investigaciones, es decir, la comprensión de la comunicación y sus impactos; y, con los derechos está la humanización y los límites de todo lo anterior.

Así, no sorprende que también a mediados del siglo XX, las naciones del mundo occidental redactaran la Declaración Universal de los Derechos Humanos, después de la segunda guerra mundial que tuvo como herramienta de control de la población a la comunicación de masas. Y en los sesenta, también se empieza a esbozar a la comunicación como un derecho humano, un hecho que profundiza el mandato del artículo 19 de la Declaración Universal y que responde a la reacción y presión que ejercían los países pobres para detener la arremetida informativa de los países desarrollados.

"La demanda por el nuevo derecho humano a la comunicación formó parte de una serie de propuestas reivindicativas que formuló principalmente el Movimiento de los Países No Alineados, en los foros del sistema de Naciones Unidas y especialmente en la Unesco. Tal demanda fue principalmente sustentada en la necesidad de proporcionar soluciones jurídicas a los problemas socioestructurales de la comunicación, en tanto ellos limitan o pueden limitar el desarrollo de los países y las personas consideradas pobres o empobrecidas, amenazan sus culturas originarias y aumentan su dependencia respecto de los países desarrollados"(Jurado: 2009).

El Informe MacBride, conocido como Un solo mundo, voces múltiples, fue un documento publicado por la Unesco en 1980, como un intento de dar una respuesta a los países del llamado Tercer Mundo que cuestionaban las formas y los fondos de la comunicación. En los sesenta, los debates internacionales sobre los problemas de la comunicación habían alcanzado puntos de confrontación estridente en muchas zonas.

Las protestas del Tercer Mundo contra el movimiento dominante de las noticias provenientes de los países industrializados se veían a menudo como ataques a la libre corriente de la información. Se afirmaba que los defensores de la libertad de prensa violaban la soberanía nacional. Se disputaba ampliamente sobre diversos conceptos de los valores de las noticias y del papel, los derechos y las responsabilidades de los periodistas, así como la contribución potencial de los medios de informativos a la solución de los grandes problemas mundiales" (MacBride, 1980: 17-18).

Sin embargo, la publicación de este informe fue debut y despedida de la Unesco en su intento de que las naciones de Occidente aceptaran a la comunicación como un derecho. La presión de los países del primer mundo, en particular del Gobierno del presidente de EE.UU., Ronald Reagan, apuntaban a que las recomendaciones ponían en riesgo la libertad de expresión, no obstante, no eran otra cosa que la oposición a que los países que no fueran parte del desarrollo, accedieran a las nuevas tecnologías de la información.

23 años después del Informe MacBride, se realizó la Cumbre Mundial de la Sociedad de la Información, como un foro en el que participaron organizaciones internacionales, los gobiernos, el sector privado y la sociedad civil para analizar el estado de la información y la comunicación, y las formas de enfrentar la desigualdad en el acceso a la información y en la brecha digital (Unesco, 2005). 
En la Cumbre, fue la sociedad civil la que intentó insertar a la comunicación como un derecho, y aunque no lo logró, se considera que hubo avances importantes: "Se promovió la idea de que es necesario tomar distancia de la expresión derecho a la comunicación o derecho a comunicar, porque ella está asociada a una mala interpretación del NOMIC -Nuevo Orden Mundial de la Información y Comunicación- (en el sentido de equivocada y maliciosamente distorsionada por sus detractores), lo cual genera muchas resistencias para reintroducir el tema en las discusiones oficiales.

Por otra parte, se señaló la conveniencia de usar la expresión derechos a la comunicación para referirse a un conjunto de derechos humanos ya existentes, pero que no estaban siendo plenamente aplicados" (Jurado, 2009).

En Ecuador, la comunicación es un derecho constitucional desde 1998. No obstante, como derecho humano integral se desarrolla en la Constitución de 2008, hasta entonces, estaba normado el derecho a acceder a información objetiva, veraz, oportuna, y a poner límites a la información reservada, fortaleciendo la transparencia del estado con la participación de la ciudadanía.

El gran salto del 2008, además de lo que señalaba la normativa del 1998, se puede definir en tres ejes: el primero relacionado con el acceso y la inclusión y, por tanto, el derecho a la comunicación que tiene enfoques sobre la diversidad, interculturalidad, la lucha contra la discriminación y el acceso a las nuevas tecnologías (artículo 16 de la Constitución). El segundo eje está relacionado al acceso a un medio de comunicación: la Constitución de 2008 promueve que cualquier ciudadano o colectivo puedan tener un medio de comunicación público, privado o comunitario, y frena el oligopolio y monopolio sobre la propiedad de los medios masivos (artículo 17). Finalmente, el tercer eje del derecho a la comunicación se concentra en el tipo, calidad y forma en que la sociedad recibe y busca información (artículo 18 de la Constitución).

Esta normativa pretendió reforzarse con la Ley Orgánica de Comunicación que entró en vigencia el 25 de junio de 2013. Esta fecha cierra la primera parte de un enfrentamiento, que duró al menos cinco años, entre los medios de comunicación privados con mayor presencia nacional, que han recibido el apoyo de sectores políticos y empresariales opuestos al régimen, con el Gobierno del presidente Rafael Correa y los medios públicos creados precisamente en este Gobierno, más otros medios privados de menor alcance, algunos sectores sociales y también políticos.

La segunda parte del enfrentamiento empezó al siguiente día de la vigencia de la Ley y apunta, por parte de los medios, a la presión internacional y a campañas internas para cuestionar lo que ellos consideran excesos y abusos que violan derechos ciudadanos por parte del régimen. Mientras que el Gobierno defiende la ley que precisamente, dice, protege a la ciudadanía de la manipulación y el engaño mediático que responde a intereses económicos concretos de las fuerzas que han gobernado para sí el país desde hace varias décadas.

La Ley de Comunicación tiene dos figuras, entre otras, muy novedosas y que han generado polémica: la primera es considerar a la comunicación, además de un derecho ciudadano sobre el que no hay discusión, un bien público, al ser un bien público el Estado está obligado a protegerlo y, por tanto, a intervenir sobre quienes 
proveen ese bien. Artículo 71 de la Ley: "La información es un derecho constitucional y un bien público; y la comunicación social que se realiza a través de los medios de comunicación es un servicio público que deberá ser prestado con responsabilidad y calidad, respetando los derechos de la comunicación establecidos en la Constitución, los instrumentos internacionales y contribuyendo al buen vivir de las personas".

La segunda novedad es la figura del linchamiento mediático que básicamente limita las publicaciones en cadena, a menudo sistemáticas, sobre las personas, en especial, sobre funcionarios públicos cuyas acciones son consideradas irregulares o simplemente sospechosas desde la perspectiva mediática. Artículo 26: "Queda prohibida la difusión de información que, de manera directa o a través de terceros, sea producida de forma concertada y publicada reiterativamente a través de uno o más medios de comunicación con el propósito de desprestigiar a una persona natural o jurídica o reducir su credibilidad pública (linchamiento mediático)".

Para la regulación, la Ley creó la Superintendencia de Comunicaciones y el Consejo de Regulación, la primera instancia tiene potestad sancionadora y la segunda reguladora. Se puede considerar que ambas entidades van a poner fin al supuesto abuso de los medios, no obstante, revelan un problema complejo: el riesgo de discrecionalidad con la que pueden actuar al evaluar qué información y programación infringe la normativa. Para cubrirse, las dos entidades han conformado equipos técnicos, especializados en comunicación y derecho, sin embargo, es tal la complejidad del tema que por la enorme carga subjetiva que tiene dicho trabajo.

No obstante, más allá de esta aspiración ideal de la vigilancia de los derechos, hay dos visiones que chocan: la perspectiva reguladora y hasta sancionadora se enfrenta con una poderosa corriente de libertades que promueve la propia Constitución.

Profundizando sobre el centro del conflicto, es posible sostener que no radica en los programas y noticias en general, ni siquiera en aquellos temas sobre los que no hay discusión en cuanto a su contenido atentatorio a diferentes derechos, sino a las secciones y espacios políticos, es decir, a la difusión de noticias, entrevistas, reportajes, artículos de opinión relacionados con los temas álgidos del escenario público que topan los intereses de los poderes en disputa.

Entonces, el conflicto es político y por tanto ideológico. Las fuerzas se enfrentan en la difusión de la información, porque de ello depende su permanencia en los distintos espacios de poder desde donde, finalmente, se trata de mantener la credibilidad social para sostenerse. En este escenario vuelve el tema de la comunicación como un servicio público para ser colocada en el rango constitucional, según la propuesta de enmienda planteada por el Gobierno Nacional: “Artículo 16.- En el artículo 384, agréguese como primer inciso el siguiente texto: La comunicación como un servicio público se prestará a través de medios públicos, privados y comunitarios”.

No obstante, la comunicación en sí misma no puede ser un bien público porque el derecho a ejercerla corre demasiados riesgos y porque materialmente es imposible que lo sea. Se confunde comunicación con información, el derecho se pone en el mismo lugar que el producto, y se relativiza el hecho de que comunicar es todo, es cualquier acto humano que emite un mensaje mediante cualquier lenguaje: la palabra, las señas, el silencio son 
actos de comunicación. Por tanto, ¿cómo puede ser un servicio si es un hecho intrínseco de la persona? Interpretarla como un servicio puede implicar que hasta con un decreto ejecutivo se legisle sobre la comunicación.

Lo que sí es un bien público es la información que ofertan los medios masivos tradicionales (periódicos, radio y televisión) o cualquier tipo de medios (redes sociales), y aquí está el meollo del asunto. Los ciudadanos se forman una opinión sobre el gobierno, los políticos, la economía, el clima, la farándula, etc., mediante la información que reciben a través de los medios masivos.

Los medios son el principal recurso de la ciudadanía para tener información de la realidad nacional, suponiendo que se pueda describir la realidad o gran parte de ella con todas las condiciones que demanda la Ley. Por ello, la información es clave y también quien la provee así como la forma de hacerlo, porque en definitiva se trata de un ejercicio de poder y lo que está detrás es la intención del control sobre lo que se publica. 


\section{Bibliografía}

Mattelart, A. y Mattelart, M (1997): Historia de las teorías de la Comunicación, Paidos, Buenos Aires.

Jurado, R. (200): Derecho a la Comunicación en Ecuador: avances, dificultades y expectativas, Quito.

Unesco, (1980): Informe MacBride.

Constitución de la República del Ecuador (2008).

Ley de Comunicación Ecuador (2013) 
